\title{
Acute kidney injury in critically ill cirrhotic patients with spontaneous bacterial peritonitis: a comparison of KDIGO and ICA criteria
}

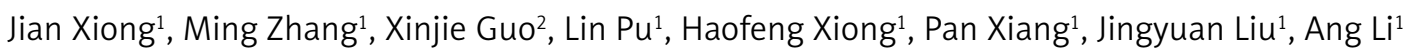

${ }^{1}$ Department of Critical Care Medicine, Beijing Ditan Hospital, Capital Medical University, Beijing, China

${ }^{2}$ Department of Critical Care Medicine, Beijing Friendship Hospital, Capital Medical

University, Beijing, China

Submitted: 29 October 2018

Accepted: 16 December 2018

Arch Med Sci 2020; 16 (3): 569-576

DOI: https://doi.org/10.5114/aoms.2019.85148

Copyright $\odot 2019$ Termedia \& Banach

\section{Abstract}

Introduction: Acute kidney injury (AKI) is an important independent predictor of mortality in cirrhotic patients with spontaneous bacterial peritonitis (SBP). However, the definition of AKI in cirrhosis has been debated for many years. This study aims to compare the prediction accuracy of Kidney Disease: Improving Global Outcomes (KDIGO) and International Club of Ascites (ICA) criteria for hospital mortality in cirrhotic patients with SBP admitted to the intensive care unit (ICU).

Material and methods: Two hundred and sixteen cirrhotic patients with SBP consecutively admitted to the ICU during 2010-2017 were retrospectively analyzed. Demographic parameters and clinical variables were collected with case report forms. Risk factors for hospital mortality were identified through a multivariate logistic regression analysis. The predictive value of ICA and KDIGO criteria was analyzed by the area under the receiver operating characteristic curve (AUROC). The primary endpoint was hospital mortality.

Results: Overall hospital mortality in our population was $73.6 \%$. Incidence of AKI was $83.8 \%$ and $81.5 \%$ according to the KDIGO and ICA classifications respectively, associated with increased in-hospital and 180-day mortality. The AKI was an independent risk factor for hospital mortality. The risk factor of AKI according to KDIGO was greater than that of ICA. The AUROC for in-hospital mortality for ICA and KDIGO was 0.730 and 0.752 , respectively. However, the predictive ability of ICA criteria for in-hospital mortality was non-inferior to that of KDIGO criteria $(p=0.123)$.

Conclusions: Both ICA and KDIGO criteria were good tools with excellent prediction performance for hospital mortality in cirrhotic patients with SBP admitted to the ICU.

Key words: cirrhosis, spontaneous bacterial peritonitis, acute kidney injury, mortality.

\section{Introduction}

Spontaneous bacterial peritonitis (SBP) is a common and life-threatening complication in patients with cirrhosis, associated with significant morbidity and mortality [1]. The hospital mortality in hospitalized patients with cirrhosis and SBP ranges from $20 \%$ to $40 \%$ in different investigations [2-5]. Due to their poor prognosis, patients with cirrhosis and SBP often need to be admitted to the intensive care unit (ICU). However, the mor-

\author{
Corresponding author: \\ Ang Li \\ Jingyuan Liu \\ Department \\ of Critical Care Medicine \\ Beijing Ditan Hospital \\ Capital Medical University \\ 8 Jingshundong St \\ Chaoyang District \\ Beijing 100015, China \\ E-mail: liang1231_bjdth@ \\ outlook.com, \\ dtyyicu@ccmu.edu.cn
}


tality in ICU-admitted cirrhotic patients with SBP has seldom been reported. The authors observed that the in-hospital and 100-day mortality in ICUadmitted cirrhotic patients with SBP were 43\% and $99 \%$ respectively [6]. Given the unacceptably high mortality in patients with cirrhosis and SBP admitted to the ICU, there is an urgent need for accurate methods of prognosis assessment and risk stratification.

Acute kidney injury $(A K I)$ is very frequent in cirrhotic patients with SBP, with a good predictive value for in-hospital mortality [2]. Moreover, a systematic review including 18 articles demonstrated that renal dysfunction is the most important independent predictor of mortality in cirrhotic patients with SBP [7]. However, the definition of AKI in cirrhosis was debated for many years until the International Club of Ascites (ICA) approached a consensus definition for the diagnosis of AKI in patients with cirrhosis in 2015 [8]. Compared with the previous Kidney Disease: Improving Global Outcomes (KDIGO) criteria [9], the use of urine output was removed in the new ICA criteria, since cirrhotic patients were often oliguric with avid renal sodium retention and yet may maintain a normal glomerular filtration rate (GFR) [10] and urine output documentation was often inconsistent and inaccurate in regular wards. To our knowledge, the prediction accuracy of the new ICA criteria and its comparison with the KDIGO criteria have never been evaluated in ICU-admitted cirrhotic patients with SBP.

The aims of this study were to determine the incidence of AKI in critically ill cirrhotic patients with SBP according to the KDIGO and ICA criteria and to compare their prediction accuracy for hospital mortality.

\section{Material and methods}

\section{Patient information and data collection}

This investigation was conducted in a 20-bed general ICU at an 800-bed university hospital in China. In this study, we included 216 consecutive

Table I. Characteristics of secondary bacterial peritonitis [14]

Ascitic fluid analysis:

- Absolute neutrophil count $>250$ cells $/ \mathrm{ml}$

- Multiple organisms on gram stain and culture

- Runyon's criteria (at least 2 of the following findings): total protein > $1 \mathrm{~g} / \mathrm{dl}$; glucose $<50 \mathrm{mg} / \mathrm{dl}$; lactate dehydrogenase greater than the upper limit of normal for serum; carcinoembryonic antigen $>5 \mathrm{ng} / \mathrm{ml}$ or alkaline phosphatase $>240$ units/l

Poor response to treatment:

- Repeat paracentesis after 48 h with: absolute neutrophil count > pre-treatment value; persistence of bacteria on culture.

- Persistence of fever and signs of peritonitis

Abnormal structural findings on imaging procedure patients with cirrhosis and SBP admitted to the ICU between 2010 and 2017. The criteria for ICU admission were established according to Guidelines for intensive care unit admission, discharge, and triage [11]. The following patients were excluded: 36 patients with documented chronic kidney disease, 1 patient with obstructive uropathy, 7 patients with severe cardiac-cerebral vascular events, 2 pregnant patients, and 33 patients without complete data.

Blood and urine routine test, blood biochemical test, coagulation function test, arterial blood gas analysis, chest radiograph, ultrasound examination, etc. were conducted among all the patients admitted to our ICU, and urine output was recorded by nurses per hour. Retrospective data were collected with case report forms, including demographic data, primary diagnosis, underlying disease, cirrhosis-related complications, biochemical analysis, Acute Physiology Age Chronic Health Evaluation II (APACHE II); Sequential Organ Failure Assessment (SOFA), Model for End-stage Liver Disease (MELD), and Child-Pugh score at the first day of ICU admission, duration of hospitalization, and in-hospital mortality. 180-day mortality was acquired from medical records or telephone interview.

\section{Definitions}

Diagnosis of cirrhosis was based on past medical history, radiological evidence of liver nodularity, endoscopic signs of portal hypertension, or liver biopsy [12]. Diagnosis of SBP was based on the presence of ascites fluid, absolute neutrophil count $>250$ cells/ $\mathrm{ml}[13]$ and the absence of features suggestive of secondary bacterial peritonitis (Table I [14]). Sepsis was defined as an acute change in SOFA score $\geq 2$ points attributed to the infection [15]. Hepatic encephalopathy was defined according to the West Haven criteria [16]. Diagnosis of pneumonia was based on radiographic finding of pulmonary infiltration associated with relevant clinical signs (fever, cough, dyspnea, or purulent sputum).

The AKI was defined and classified according to ICA and KDIGO criteria (Table II). The peak AKI stage during the ICU stay was used. The last serum creatinine within the previous 3 months before hospitalization was used as the baseline serum creatinine. In patients without previous serum creatinine, the first serum creatinine during hospitalization was used $[8,17]$. A predictive model for hospital mortality was applied as follows: non-AKI (0 points); ICA stage 1, and KDIGO stage 1 (1 point); ICA stage 2 , and KDIGO stage 2 (2 points); ICA stage 3 , and KDIGO stage 3 (3 points).

\section{Ethics approval and consent to participate}

This clinical study was conducted in compliance with the ethical principles of the Declaration of Helsinki and its later amendments. The Ethics 
Table II. ICA and KDIGO classification for AKI

\begin{tabular}{|c|c|c|}
\hline Parameter & $\mathrm{SCr}$ & uO \\
\hline \multicolumn{3}{|l|}{ ICA: } \\
\hline Definition & $\begin{array}{c}\text { Increase in } \mathrm{sCr} \geq 0.3 \mathrm{mg} / \mathrm{dl} \text { within } 48 \mathrm{~h} \text {; or a percentage increase } \\
\mathrm{s} C \mathrm{Cr} \geq 1.5 \text {-fold from baseline which is known, or presumed, to have } \\
\text { occurred within the prior } 7 \text { days }\end{array}$ & Not provided \\
\hline Stage 1 & $\begin{array}{l}\text { Increase in } \mathrm{sCr} \geq 0.3 \mathrm{mg} / \mathrm{dl} \text { or an increase in } \mathrm{s} C \mathrm{r} \geq 1.5 \text {-fold to } 2 \text {-fold } \\
\text { from baseline }\end{array}$ & Not provided \\
\hline Stage 2 & Increase in $\mathrm{s} C \mathrm{r} \geq 2$-fold to 3 -fold from baseline & Not provided \\
\hline Stage 3 & $\begin{array}{c}\text { Increase in } \mathrm{s} C r \geq 3 \text {-fold from baseline or } \mathrm{s} C r \geq 4.0 \mathrm{mg} / \mathrm{dl} \text { with } \\
\text { an acute increase } \geq 0.3 \mathrm{mg} / \mathrm{dl} \text { or initiation of renal replacement } \\
\text { therapy }\end{array}$ & Not provided \\
\hline \multicolumn{3}{|l|}{ KDIGO: } \\
\hline Definition & $\begin{array}{l}\text { Increase in } \mathrm{s} C r \text { by } \geq 0.3 \mathrm{mg} / \mathrm{dl} \text { within } 48 \mathrm{~h} \text {; or increase in } \mathrm{s} C \mathrm{r} \geq 1.5 \\
\text { times baseline to have occurred within the prior } 7 \text { days }\end{array}$ & $<0.5 \mathrm{ml} / \mathrm{kg} / \mathrm{h}$ for $6 \mathrm{~h}$ \\
\hline Stage 1 & $1.5-1.9$ times baseline; or $\geq 0.3 \mathrm{mg} / \mathrm{dl}$ increase & $<0.5 \mathrm{ml} / \mathrm{kg} / \mathrm{h}$ for $6-12 \mathrm{~h}$ \\
\hline Stage 2 & 2.0-2.9 times baseline & $<0.5 \mathrm{ml} / \mathrm{kg} / \mathrm{h}$ for $\geq 12 \mathrm{~h}$ \\
\hline Stage 3 & $\begin{array}{c}\text { 3.0 times baseline; or increase in } \mathrm{s} C \mathrm{r} \text { to } \geq 4.0 \mathrm{mg} / \mathrm{dl} \text {; or initiation of } \\
\text { renal replacement therapy }\end{array}$ & $\begin{array}{l}<0.3 \mathrm{ml} / \mathrm{kg} / \mathrm{h} \text { for } \geq 24 \mathrm{~h} \\
\quad \text { or anuria for } \geq 12 \mathrm{~h}\end{array}$ \\
\hline
\end{tabular}

ICA - International Club of Ascites, KDIGO - Kidney Disease: Improving Global Outcomes, sCr - serum creatinine, UO - urine output. The worst possible classification of serum creatinine or urine output criteria result was used.

Committee of Beijing Ditan Hospital approved our study protocol (approval no. KY2016-022). As a de-identified retrospective study, the ethical committee did not require any written or verbal informed consent from participants.

\section{Statistical analysis}

Normally distributed variables were presented as means and standard deviations while non-normally distributed variables were presented as medians with interquartile ranges. Normality was tested by the Kolmogorov-Smirnov test. Normally distributed variables were compared by Student's $t$ test and non-normally distributed variables were compared by the Kruskal-Wallis test. Categorical variables were presented with proportions and compared by the $\chi^{2}$. Risk factors associated with in-hospital mortality were identified through multivariate logistic regression analysis. Predictive accuracy was assessed by the area under the receiver operating characteristic curve (AUROC). A nonparametric approach was used to compare the areas under the two ROC curves [18]. The Kaplan-Meier approach was used to determine survival curves, and a log-rank test was employed to compare the differences between the curves. Data were analyzed using SPSS 19.0 for Windows (SPSS, Chicago, IL, USA), and a $p$-value less than 0.05 was considered statistically significant.

\section{Results}

\section{Subject characteristics}

Two hundred and sixteen patients with cirrhosis admitted to the ICU during 2010-2017 were enrolled in this investigation. Their mean age was 57.6 years; 174 (80.6\%) patients were male. The overall in-hospital mortality was $73.6 \%$ (159/216), and the 180-day mortality was $87.0 \%$ (188/216). Table III presents the clinical characteristics of survivors and non-survivors. Survivors and non-survivors were similar in age, sex, weight, causes of cirrhosis, alanine aminotransferase, hypertension, diabetes, hepatic encephalopathy, upper gastrointestinal bleeding and pneumonia. Non-survivors had higher serum creatinine, bilirubin, international normalized ratio (INR), SOFA, APACHE II, MELD, Child-Pugh score, lower serum sodium, albumin and shorter lengths of ICU and hospital stay. A higher proportion of non-survivors had sepsis. Liver disease was mostly attributed to hepatitis B viral infection (42.6\%).

\section{Comparison of AKI incidence according to the ICA and KDIGO criteria}

Number of patients classified into the respective stages of AKI by ICA and KDIGO were cross-tabulated against each other (Table IV). The incidence of AKI was $81.5 \%$ according to ICA criteria, and $83.8 \%$ according to KDIGO criteria. One hundred and ninety-four (89.8\%) of 216 patients had the same degree of AKI according to both classification systems. There was a significant correlation between ICA and KDIGO classification (Spearman rank correlation coefficients: $r=0.929, p<0.001)$.

\section{In-hospital mortality of patients according to ICA and KDIGO classification}

The AKI was an independent risk factor for hospital mortality, irrespective of which criteria 
Jian Xiong, Ming Zhang, Xinjie Guo, Lin Pu, Haofeng Xiong, Pan Xiang, Jingyuan Liu, Ang Li

Table III. Demographic data and clinical characteristics of survivors and non-survivors

\begin{tabular}{|c|c|c|c|c|}
\hline Parameter & $\begin{array}{l}\text { All patients } \\
(n=216)\end{array}$ & $\begin{array}{l}\text { Survivors } \\
(n=57)\end{array}$ & $\begin{array}{c}\text { Non-survivors } \\
\quad(n=159)\end{array}$ & $P$-value \\
\hline Age [years] & $57.6 \pm 12.9$ & $57.9 \pm 14.7$ & $57.5 \pm 12.3$ & 0.816 \\
\hline Male, $n(\%)$ & $174(80.6)$ & $50(87.7)$ & $124(78.0)$ & 0.111 \\
\hline Weight [kg] & $67.0(59.4-72.9)$ & $66.0(59.3-70.9)$ & $67.2(59.4-74.0)$ & 0.400 \\
\hline Lengths of ICU stay [days] & $5(2-10)$ & $8(5-17)$ & $4(2-9)$ & $<0.001$ \\
\hline Lengths of hospital stay [days] & $14(6-27)$ & $26(15-34)$ & $10(5-22)$ & $<0.001$ \\
\hline Causes of cirrhosis, $n(\%)$ : & & & & 0.697 \\
\hline Alcoholic & $56(25.9)$ & $18(31.6)$ & $38(23.9)$ & \\
\hline Hepatitis B & $92(42.6)$ & $24(42.1)$ & $68(42.8)$ & \\
\hline Hepatitis C & $7(3.2)$ & $2(3.5)$ & $5(3.1)$ & \\
\hline Autoimmune & $18(8.3)$ & $6(10.5)$ & $12(7.5)$ & \\
\hline Mixed etiology & $22(10.2)$ & $3(5.3)$ & $19(11.9)$ & \\
\hline Unknown etiology & $16(7.4)$ & $3(5.3)$ & $13(8.2)$ & \\
\hline Other causes* & $5(2.3)$ & $1(1.8)$ & $4(2.5)$ & \\
\hline Serum creatinine $[\mu \mathrm{mol} / \mathrm{l}]$ & $143.9(82.7-244.3)$ & $99.7(67.3-144.9)$ & $162.3(108.9-264.6)$ & $<0.001$ \\
\hline Serum sodium [mmol/l] & $132.4 \pm 8.0$ & $134.5 \pm 7.7$ & $131.6 \pm 8.1$ & 0.021 \\
\hline Albumin $[\mathrm{g} / \mathrm{l}]$ & $27.9(24.9-31.5)$ & $29.4(25.8-33.4)$ & $27.6(24.7-30.4)$ & 0.015 \\
\hline Bilirubin $[\mu \mathrm{mol} / \mathrm{l}]$ & $109.5(40.8-306.6)$ & $51.7(27.5-204.9)$ & $128.4(55.1-369.1)$ & 0.002 \\
\hline ALT [units/l] & $41.6(20.0-92.4)$ & $33.3(17.4-72.7)$ & $46.6(20.1-101.1)$ & 0.093 \\
\hline INR & $1.94(1.57-2.88)$ & $1.66(1.39-2.07)$ & $2.17(1.62-3.07)$ & $<0.001$ \\
\hline Diabetes, $n(\%)$ & $64(29.6)$ & $17(29.8)$ & $47(29.6)$ & 0.970 \\
\hline Hypertension, $n$ (\%) & $61(28.2)$ & $17(29.8)$ & $44(27.7)$ & 0.757 \\
\hline $\mathrm{HE}, n(\%)$ & $143(66.2)$ & $34(59.6)$ & $109(68.6)$ & 0.223 \\
\hline UGIB, $n(\%)$ & $83(38.4)$ & $19(33.3)$ & $64(40.3)$ & 0.357 \\
\hline Sepsis, $n$ (\%) & $117(54.2)$ & $21(36.8)$ & $96(60.4)$ & 0.002 \\
\hline Pneumonia, $n$ (\%) & $93(43.1)$ & $24(42.1)$ & $69(43.4)$ & 0.866 \\
\hline MELD & $24 \pm 12$ & $16 \pm 10$ & $27 \pm 11$ & $<0.001$ \\
\hline SOFA & $11(8-14)$ & $9(7-11)$ & $12(9-15)$ & $<0.001$ \\
\hline APACHE II & $21 \pm 8$ & $17 \pm 7$ & $22 \pm 8$ & $<0.001$ \\
\hline Child-Pugh score & $13(11-14)$ & $11(10-13)$ & $13(12-14)$ & $<0.001$ \\
\hline
\end{tabular}

ICU - intensive care unit, ALT - alanine aminotransferase, INR - international normalized ratio, HE - hepatic encephalopathy, UGIB upper gastrointestinal bleeding, MELD - model for end-stage liver disease, SOFA - sequential organ failure assesSment, APACHE - acute physiology and chronic health evaluation; *hepatolenticular degeneration, schistosomiasis cirrhosis, cardiac cirrhosis.

were applied. However, AKI stage 1 defined by ICA criteria was not an independent risk factor for hospital mortality. The odds ratios of AKI stages in predicting hospital mortality according to the ICA and KDIGO classifications are listed in Table V. The odds ratio for hospital mortality of AKI stage 3 was significantly higher than the other stages. There was a progressive increase in the mortality rate correlated with the increasing AKI stage. However, there was no significant difference in hospital mortality between patients in AKI stages 2 and 3 for ICA criteria. The increase in odds ratio with increasing stages of AKI was greater in the KDIGO criteria. Figures 1 and 2 show a significant difference in the 180-day cumulative survival rates between patients with and without AKI, regardless of the applied AKI criteria. However, the 180-day cumulative survival rates were similar between AKI stages 2 and 3 when the ICA criteria were used (Figure 1). 
Table IV. Cross tabulation of patients classified by ICA versus KDIGO

\begin{tabular}{|lccccc|}
\hline \multirow{2}{*}{ KDIGO } & \multicolumn{3}{c}{ ICA } & Total \\
\cline { 2 - 5 } & Non-AKI & Stage 1 & Stage 2 & Stage 3 & \\
\hline Non-AKI & $35(16.2 \%)$ & 0 & 0 & 0 & $35(16.2 \%)$ \\
\hline Stage 1 & $2(0.9 \%)$ & $28(13.0 \%)$ & 0 & 0 & $30(13.9 \%)$ \\
\hline Stage 2 & $2(0.9 \%)$ & $6(2.8 \%)$ & $24(11.1 \%)$ & 0 & $32(14.8 \%)$ \\
\hline Stage 3 & $1(0.5 \%)$ & $3(1.4 \%)$ & $8(3.7 \%)$ & $107(49.5 \%)$ & $119(55.1 \%)$ \\
\hline Total & $40(18.5 \%)$ & $37(17.1 \%)$ & $32(14.8 \%)$ & $107(49.5 \%)$ & $216(100 \%)$ \\
\hline
\end{tabular}

AKI - acute kidney injury, ICA - International Club of Ascites, KDIGO - Kidney Disease: Improving Global Outcomes.

Table V. Odds ratio of AKI stages in predicting hospital mortality by multivariable logistic regression

\begin{tabular}{|c|c|c|c|c|}
\hline Score & $N$ & In-hospital mortality (\%) & OR $(95 \% \mathrm{Cl})$ & $P$-value \\
\hline \multicolumn{5}{|l|}{ ICA: } \\
\hline ICA-O & 40 & 40.0 & 1 (reference) & - \\
\hline ICA-1 & 37 & 64.9 & $2.461(0.818-7.406)$ & 0.109 \\
\hline ICA-2 & 32 & 81.3 & $3.752(1.067-13.193)$ & 0.039 \\
\hline ICA-3 & 107 & 86.9 & $7.170(2.321-22.149)$ & 0.001 \\
\hline \multicolumn{5}{|l|}{ KDIGO: } \\
\hline KDIGO-O & 35 & 34.3 & 1 (reference) & - \\
\hline KDIGO-1 & 30 & 63.3 & $3.580(1.075-11.927)$ & 0.038 \\
\hline KDIGO-2 & 32 & 71.9 & $3.693(1.074-12.694)$ & 0.038 \\
\hline KDIGO-3 & 119 & 88.2 & $10.955(3.246-36.968)$ & $<0.001$ \\
\hline
\end{tabular}

ICA - International Club of Ascites, KDIGO - Kidney Disease: Improving Global Outcomes, OR - odds ratio.

Comparison of calibration and discrimination for predicting hospital mortality

Discrimination between the ICA and KDIGO criteria in predicting hospital mortality is shown in Figure 3. The results of goodness-of-fit mea-

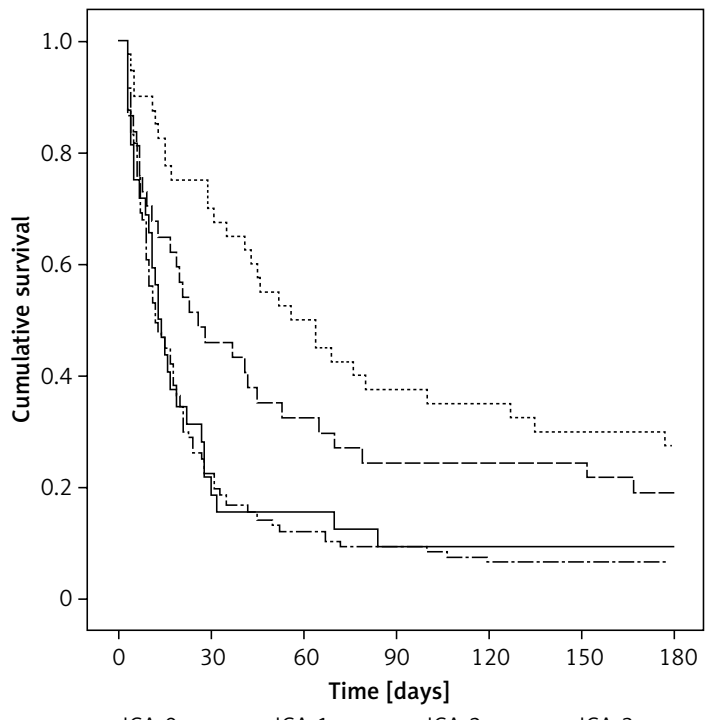

… ICA-0 -- ICA-1 —ICA-2 --ICA-3

Figure 1. 180-day survival curves of patients according to the ICA classification

ICA - International Club of Ascites. sured by Hosmer-Lemeshow $\chi^{2}$ statistics denoted the predictive accuracy of the ICA $(p=0.688)$ and KDIGO $(p=0.798)$ criteria (Table VI). The AUROC values for in-hospital mortality of ICA and KDIGO criteria were 0.730 and 0.752 respectively, but were not statistically significant $(p=0.123)$.

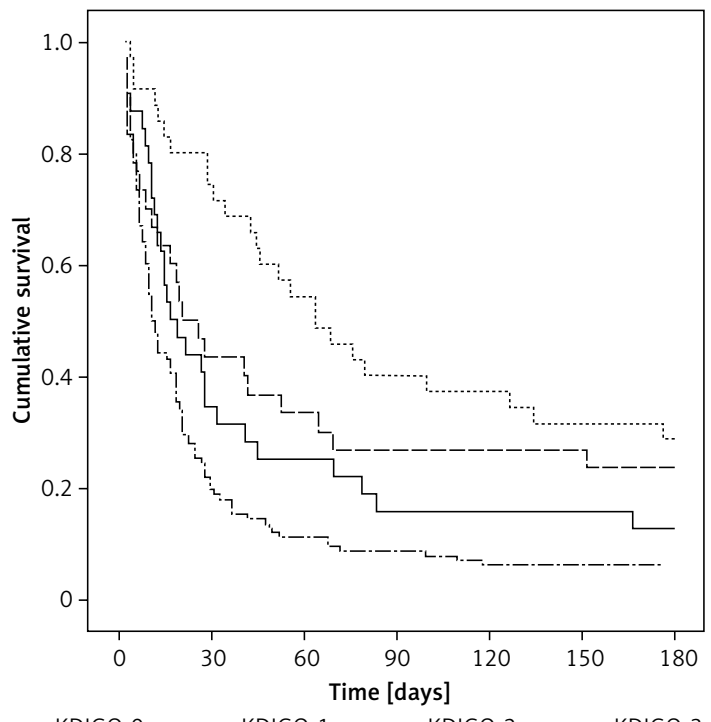

-.... KDIGO-0 --- KDIGO-1 - KDIGO-2 -.- KDIGO-3

Figure 2. 180-day survival curves of patients according to the KDIGO classification

KDIGO - Kidney Disease: Improving Global Outcomes. 


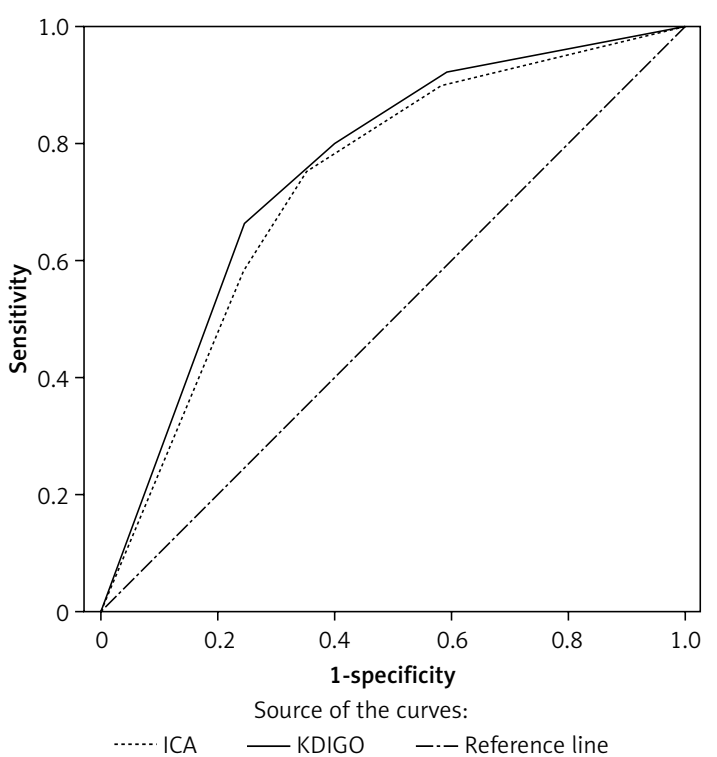

Figure 3. Receiver operating characteristics curves for in-hospital mortality according to KDIGO and ICA classification

KDIGO - Kidney Disease: Improving Global Outcomes, ICA - International Club of Ascites, KDIGO - AUROC $=0.752$ (95\% Cl: $0.674-0.830, p<0.001) ;$ ICA: $A U R O C=0.730$ (95\% Cl: $0.649-0.810, p<0.001) .{ }^{*} P=0.123$, KDIGO vs. ICA.

\section{Discussion}

This study provides some information about the prevalence and prognosis of $\mathrm{AKI}$ in ICU-admitted cirrhotic patients with SBP. AKI was shown to be an independent risk factor for in-hospital mortality, regardless of the ICA or KDIGO criteria. The presence and severity of AKI were associated with a significantly graded risk of mortality in patients with cirrhosis and SBP admitted to the ICU. Both ICA and KDIGO criteria had a good predictive ability for hospital mortality in ICU-admitted cirrhotic patients with SBP.

Previous studies demonstrated that approximately $30 \%$ of cirrhotic patients with SBP presented with renal impairment $[19,20]$. However, few studies have investigated the incidence of renal impairment in those admitted to the ICU. An investigation from the Institute of Liver and Biliary Science, New Delhi, demonstrated that AKI occurred in $45.41 \%$ (99/218) of patients with cirrhosis and SBP admitted to the ICU [6]. In our study, the incidence of AKI was $81.5 \%$ according to ICA criteria, and $83.8 \%$ according to KDIGO criteria, which was significantly higher than that of the previous investigations. The difference of AKI prevalence may be related to the diversity of AKI definitions and different clinical settings. Furthermore, the investigation from New Delhi only considered the baseline AKI level at ICU admission, and the AKI incidence would have been much higher if the patients who acquired AKI during the ICU stay had been included as well. In all, AKI occurs in about 19\% (293/1544) of patients with cirrhosis according to a review article [21]. For cirrhotic patients with SBP, the incidence of AKI increases to approximately $30 \%[19,20]$. Although there is not enough evidence, it is likely that AKI is very frequent in critically ill cirrhotic patients with SBP. This needs to be validated in further large prospective cohort studies.

Isolated serum creatinine is inaccurate in the diagnosis of renal impairment in patients with cirrhosis since they often have a low level of serum creatinine owing to the reduced hepatic production of creatinine from creatine and muscle wasting. Given the limitations of isolated serum creatinine, the ICA developed a new consensus definition for the diagnosis of AKI in patients with cirrhosis based on KDIGO criteria [8]. However, the urine output was removed in the new consensus definition of AKI, which raised some arguments about the use of urine output in patients with cirrhosis. A recent study from the University of Pittsburgh reported that patients with oliguria had a nearly 3 -fold increased rate of hospital mortality compared with patients without AKI in critically ill patients with chronic liver disease [22]. In our study, the predictive ability of ICA criteria was non-inferior to that of KDIGO, in spite of removal of urine output. Although our study included a relatively small cohort of patients, the difference of AUROC between ICA and KDIGO criteria might be of statistical significance when validated in a larger cohort study.

Many studies have reported that renal impairment was an important predictor for hospital mortality in patients with cirrhosis and SBP [19, 20]. A systemic review from Yale University School of Medicine showed that renal dysfunction was the most important independent predictor of mor-

Table VI. Calibration and discrimination of the AKI scores in predicting in-hospital mortality

\begin{tabular}{|lcccccc|}
\hline Variable & \multicolumn{3}{c}{ Calibration } & \multicolumn{3}{c|}{ Discrimination } \\
\cline { 2 - 7 } & Hosmer-Lemeshow $\chi^{2}$ & df & $P$-value & AUROC \pm SE & $95 \% \mathrm{Cl}$ & $P$-value \\
\hline ICA & 0.688 & 2 & 0.709 & $0.730 \pm 0.041$ & $0.649-0.810$ & $<0.001$ \\
\hline KDIGO & 0.798 & 2 & 0.671 & $0.752 \pm 0.040^{*}$ & $0.674-0.830$ & $<0.001$ \\
\hline
\end{tabular}

ICA - International Club of Ascites, KDIGO - Kidney Disease: Improving Global Outcomes, AUROC - areas under the receiver operating characteristic curves, $S E-$ standard error, $\mathrm{Cl}$ - confidence intervals. ${ }^{*} P=0.123 \mathrm{vs}$. ICA. 
tality in cirrhotic patients with SBP [7]. de Araujo et al. validated the predictive value of AKIN (Acute Kidney Injury Network) criteria in cirrhotic patients with SBP and concluded that AKIN criteria were useful to predict mortality in patients with SBP [2]. In de Araujo's study, AKIN criteria were also performed without urine output, which was similar to the ICA criteria. Though the predictive value of AKI was also great after the removal of urine output, we still could not deny the possibility that incorporation of urine output into the AKI criteria might improve the predictive value of AKI for prognosis, something which needs to be validated in future studies.

Many investigations have reported that the hospital mortality in patients with cirrhosis and SBP was $20-40 \%$ [2-5, 23, 24]. The overall in-hospital mortality was $73.6 \%$ in our study. The prognosis in our investigation was worse than those of previous studies, which might be because those studies were conducted in regular wards. Patients admitted to the ICU suffered from more organ failures and comorbidities, which was the primary reason that the mortality was higher in our study than that of patients with SBP in regular wards. The investigation from the Institute of Liver and Biliary Science, New Delhi, reported that the 20-, $40-$, and 60-day mortalities were $83.0 \%, 95.4 \%$, and $99.5 \%$ respectively in patients with cirrhosis and SBP admitted to the ICU [6], whose prognosis was also significantly worse than that of the investigations in regular wards. Nevertheless, AKI was still an independent risk factor for hospital mortality in cirrhotic patients with SBP admitted to the ICU when confounding factors were included in the logistic mode for adjustment.

To our knowledge, our study is the first to evaluate the predictive value of the new ICA-AKI criteria for hospital mortality in patients with cirrhosis and SBP admitted to the ICU. The study showed the high incidence of AKI in ICU-admitted cirrhotic patients with SBP. On the other hand, several limitations should also be considered in our study. First, this study suffers from potential biases related to its retrospective design. Second, this investigation was performed at one academic tertiary-care medical center; the results may not be extrapolated to other centers. Finally, our conclusion may not be applicable to patients outside the ICU.

In conclusion, this study showed a high incidence of AKI in patients with cirrhosis and SBP admitted to the ICU. Critically ill cirrhotic patients with SBP presented a poor prognosis which was directly associated with the presence and severity of AKI. AKI was an independent risk factor for hospital mortality irrespective of the applied criteria. Both ICA and KDIGO criteria could predict hospital mortality in cirrhotic patients with SBP admitted to the ICU.

\section{Acknowledgments}

Jian Xiong and Ming Zhang contributed equally to this work.

The authors gratefully acknowledge Mrs. Junnan Li for her assistance with data analysis and technical graphics.

This work was supported by the Research Award Fund for Clinical Medicine of Beijing Hospital Authority, China (Grant No: ZYLX201802)

\section{Conflict of interest}

The authors declare no conflict of interest.

\section{References}

1. Jawid AWR, Subramanian IM. Spontaneous Bacterial Peritonitis. Springer International Publishing 2017.

2. de Araujo A, Alvares-Da-Silva MR. Akin criteria as a predictor of mortality in cirrhotic patients after spontaneous bacterial peritonitis. Annf Hepatol 2014; 13: 390-5.

3. Iliaz R, Ozpolat T, Baran B, et al. Predicting mortality in patients with spontaneous bacterial peritonitis using routine inflammatory and biochemical markers. Eur J Gastroenterol Hepatol 2018; 30: 786-91.

4. Terg R, Gadano A, Cartier M, et al. Serum creatinine and bilirubin predict renal failure and mortality in patients with spontaneous bacterial peritonitis: a retrospective study. Liver Int 2010; 29: 415-9.

5. Schwabl P, Bucsics T, Soucek K, et al. Risk factors for development of spontaneous bacterial peritonitis and subsequent mortality in cirrhotic patients with ascites. Liver Int 2015; 35: 2121-8.

6. Bal CK, Daman R, Bhatia V. Predictors of fifty days in-hospital mortality in decompensated cirrhosis patients with spontaneous bacterial peritonitis. World J Hepatol 2016; 8: 566-72.

7. Tandon P, Garcia-Tsao G. renal dysfunction is the most important independent predictor of mortality in cirrhotic patients with spontaneous bacterial peritonitis. Clin Gastroenterol Hepatol 2011; 9: 260-5.

8. Angeli $P$, Ginès $P$, Wong $F$, et al. Diagnosis and management of acute kidney injury in patients with cirrhosis: revised consensus recommendations of the International Club of Ascites. Gut 2015; 64: 531-7.

9. Kidney Disease: Improving Global Outcomes. Acute Kidney Injury Work Group: KDIGO clinical practice guideline for acute kidney injury. Kidney Int Suppl 2012; 2: 1-138.

10. Angeli P, Gatta A, Caregaro L, et al. Tubular site of renal sodium retention in ascitic liver cirrhosis evaluated by lithium clearance. Eur J Clin Investig 1990; 20: 111-7.

11. Egol A, Fromm R, Guntupalli K, et al. Guidelines for intensive care unit admission, discharge, and triage. Crit Care Med 1999; 27: 633-8.

12. Tsochatzis EA, Bosch J, Burroughs AK. Liver cirrhosis. Lancet 2014; 383: 1749-61.

13. Ginès $P$, Angeli $P$, Lenz $K$, et al. EASL clinical practice guidelines on the management of ascites, spontaneous bacterial peritonitis, and hepatorenal syndrome in cirrhosis. J Hepatol 2010; 53: 397-417.

14. Lu MLR, Agarwal A, Sloan J, Kosmin A. Infected ascites: distinguishing secondary peritonitis from spontaneous 
bacterial peritonitis in a cirrhotic patient with classic symptoms. IDCases 2017; 8: 29-31.

15. Singer M, Deutschman CS, Seymour CW, et al. The Third International Consensus definitions for sepsis and septic shock (Sepsis-3). JAMA 2016; 315: 762-74.

16. Riordan SM, Williams R. Treatment of hepatic encephalopathy. N Engl J Med 1997; 365: 473-9.

17. Pan H, Chien YS, Jenq CC, et al. Acute kidney injury classification for critically ill cirrhotic patients: a comparison of the KDIGO, AKIN, and RIFLE classifications. Sci Rep 2016; 6: 23022.

18. Delong ER, Delong DM, Clarkepearson DL. Comparing the areas under two or more correlated receiver operating characteristic curves: a nonparametric approach. Biometrics 1988; 44: 837-45.

19. Perdomo CG, Alves dMA. Renal impairment after spontaneous bacterial peritonitis: incidence and prognosis. Can J Gastroenterol 2003; 17: 187-90.

20. Follo A, Llovet JM, Navasa M, et al. Renal impairment after spontaneous bacterial peritonitis in cirrhosis: incidence, clinical course, predictive factors and prognosis. Hepatology 2010; 20: 1495-501.

21. Garcia-Tsao G, Parikh CR, Viola A. Acute kidney injury in cirrhosis. Hepatology 2008; 48: 2064-77.

22. Amathieu R, Al-Khafaji A, Sileanu FE, et al. Significance of oliguria in critically ill patients with chronic liver disease. Hepatology 2017; 66: 1592-600.

23. Nobre SR, Cabral JE, Gomes JJ, Leitão MC. In-hospital mortality in spontaneous bacterial peritonitis: a new predictive model. Eur J Gastroenterol Hepatol 2008; 20: 1176-81

24. Toledo C, Salmeron JM, Rimola A, et al. Spontaneous bacterial peritonitis in cirrhosis: predictive factors of infection resolution and survival in patients treated with cefotaxime. Hepatology 2010; 17: 251-7. 